FedUni ResearchOnline https://researchonline.federation.edu.au

This is the peer-reviewed version of the following article:

Kumar, S., Das, N., Islam, S. (2016) Software implementation of two seamless redundant topologies in a digital protection system based on IEC 62439-3. 2016 Australasian Universities Power Engineering Conference, AUPEC 2016; Brisbane, Australia; 25th-28th September 2016 p. 1-5.

Which has been published in final form at: https://doi.org/10.1109/AUPEC.2016.7749356

Copyright (C) 2016 IEEE. Personal use of this material is permitted. Permission from IEEE must be obtained for all other uses, in any current or future media, including reprinting/republishing this material for advertising or promotional purposes, creating new collective works, for resale or redistribution to servers or lists, or reuse of any copyrighted component of this work in other works. 


\title{
Software Implementation of Two Seamless Redundant Topologies in a Digital Protection System based on IEC 62439-3
}

\author{
Shantanu Kumar, ${ }^{1,3}$ Member, IEEE, Narottam Das, ${ }^{1,2}$ Senior Member, IEEE, and Syed Islam, ${ }^{1}$ Senior Member, IEEE \\ ${ }^{1}$ Department of Electrical and Computer Engineering, Curtin University, Perth, WA 6845, Australia \\ ${ }^{2}$ School of Mechanical and Electrical Engineering, University of Southern Queensland, Toowoomba, QLD 4350, Australia \\ ${ }^{3}$ Asset Performance Department, Western Power, Perth, WA 6000, Australia \\ e-mails: shantanu.kumar@postgrad.curtin.edu.au,narottam.das@usq.edu.au, and s.islam@curtin.edu.au
}

\begin{abstract}
Substations are the junctions of any transmission and distribution network in utilities. They carry bulk power to the customers using transmission network involving high voltage $(\mathrm{HV})$ apparatus. Protection of $\mathrm{HV}$ equipment during a fault is the primary concern of an operator. Advancement in design and manufacturing of Intelligent Electronic Devices (IEDs) and IEC 61850 are finding increased application in utilities from automation and communication perspective, which addresses few shortcomings that couldn't be resolved in a conventional system. However there are few challenges in the digital scheme such as end to end delays in the packet transfer and interoperability of devices. Utilities are able to perceive these benefits by leveraging on IEC 62439-3 which has two important topologies i.e. Parallel Redundancy Protocol (PRP) and High-availability Seamless Redundancy (HSR). It offers seamless redundancy in digital protection network without compromising the availability and downtime cost. In this paper, an Optimized Network Engineering Tool (OPNET) is used to simulate and compare the performance of these topologies with respect to delay time.
\end{abstract}

Index Terms-Ethernet, IEC 61850, IEC 62439-3, PRP and HSR.

\section{INTRODUCTION}

$M$ odern substations are expected to work in a reliable and efficient environment providing adequate redundancy in protection to the substation assets and transmission lines interconnecting these high voltage (HV) substations. Lack of redundancy in protection scheme in an event of a fault, could cause costly downtime, high starting costs and loss of valuable data. This could have enormous impact on the operator technically and financially.

Ethernets are increasingly shaping up the automation requirement and proving to be a dependable protection scheme from an automation and control perspective. Substation Automation System (SAS) which works at three layers i.e., 1) Station, 2) Bay, and 3) Process layers, brings digital technology to the fore front of a smart grid substation. However, strict performance monitoring and validation of the results are few key prerequisites prior to its implementation in mass which does not seem to be enjoying the confidence of the utility operators. Although, IEC 61850 lays down certain guidelines for communication protocol at station, bay and process level components, yet issues related to redundancy offered in the protection scheme seems to have gaps, as frames are lost at nodes and switches [1]. Furthermore, the timing requirement of a protection scheme to operate during a fault being very critical, such as interlocking and blocking, the scheme must act quickly operate within 4-ms as per the IEC 61850-5 standard which has been exhibited in Table-I. In such challenging circumstances, the redundant structure within a topology play a vital role. This is achieved by having a redundancies in the system i.e., the entire network is duplicated including the communication channel while in media redundancy, system and devices are individually duplicated.

Table-I: Communication recovery time based on IEC 61850-5 Ed. 2 [2].

\begin{tabular}{|l|c|c|}
\hline $\begin{array}{l}\text { Communication } \\
\text { Partners }\end{array}$ & $\begin{array}{l}\text { Application } \\
\text { Recovery time } \\
\text { (in ms) }\end{array}$ & $\begin{array}{l}\text { Communication } \\
\text { Recovery Time } \\
\text { (in ms) }\end{array}$ \\
\hline $\begin{array}{l}\text { Client - Server } \\
\text { SC to IED }\end{array}$ & 800 & 400 \\
\hline NTP, SNMP & 500 & 300 \\
\hline $\begin{array}{l}\text { IED to IED, } \\
\text { reverse } \\
\text { blocking, } \\
\text { interlocking }\end{array}$ & 12 & 4 \\
\hline Trip GOOSE & 8 & 4 \\
\hline $\begin{array}{l}\text { Bus Bar } \\
\text { Protection }\end{array}$ & $<1$ & seamless \\
\hline $\begin{array}{l}\text { Sampled } \\
\text { Values }\end{array}$ & $<2$ & 0 \\
\hline
\end{tabular}

In a recent development, IEC TC57 working group in 2010 have used IEC 62439-3 Ed. 1 and 2 standards to address the redundancy issues in digital protection of a SAS network. Leveraging on IEC 62439-3 standard, PRP and HSR topologies provide seamless recovery in case of a single link failure. IEC 62439-3, Edition 2 published in 2012 has undertaken few changes with respect to:-

- Tagging of PRP frames

- Clarification on interoperability

- Consideration of clock synchronization

- Simplifying the testing and commissioning procedure.

It may be noted, in a PRP topology, Doubly Attached Node (DAN) enabled equipment are connected as shown in Fig. 1 
with frames circulated in two parallel networks. This is layer 2 redundancy with equipment connected in a cross structured linked engineering environment. It fully supports GOOSE and Sampled Values (SV) traffic in a process bus structure.

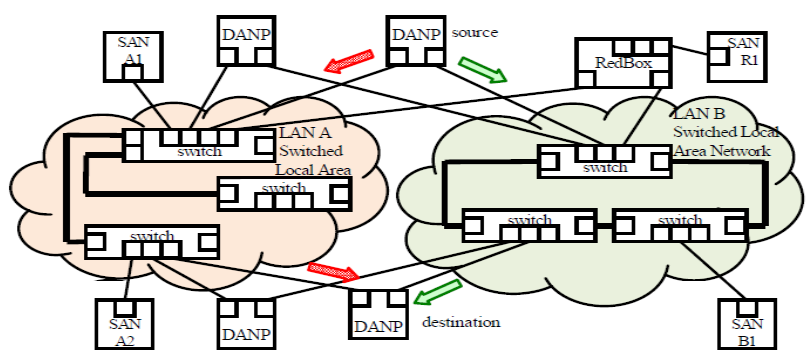

Fig. 1: PRP Network in a digital protection scheme [1].

Fig. 2 shows a typical HSR network in a ring topology wherein IEDs are connected in DAN with two independent paths for frame circulation. In this topology the source sends two frames in two opposite direction ensuring at least one frame reach its destination.

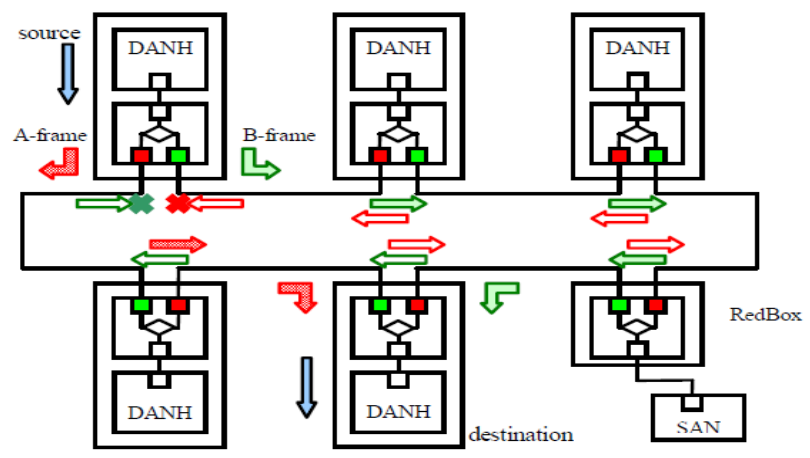

Fig. 2: HSR Topology in a digital protection scheme [1].

In both PRP and HSR topologies, Link Redundancy Entity (LRE) which buffers between ports and upper layer, manages the frame traffic transition. Substation IEDs with a Single Attached Node (SAN) which needs to have PRP and HSR features that must be connected via Red Box has been exhibited in Fig. 1 and Fig. 2[1]. By introducing Red boxes in PRP/HSR topologies, standard SAN IED devices can be converted to DAN devices and redundancy in the digital network could be achieved. In this scheme, one host port is connected to the standard digital protection device while two other ports are meant for redundancies.

As per IEC 62439-3 guideline, SAS scheme must regularly monitor and check the frames at an interval per minute scanning the entire network for SV and GOOSE packets checking for the loss of the missing frames. IEC 62439-3 not only checks for redundancies in the network, but also at the nodes and other compliant devices connected in the topology. Although the initial capital cost of PRP topology in a digital protection scheme is higher than an equivalent conventional one due to the duplication of protection infrastructure, yet it offers reliability and redundancy which could negate other disadvantages. Further, manufacturers are working towards upgrading Rapid Spanning Tree Protocol (RSTP) devices interfacing with Red boxes which makes it fully compliant to IEC 62439-3 standard.
However, there are very few utilities around the world which are operational using PRP/HSR digital protection topologies.

The PRP and HSR network have few differences such as; PRP manages two independent networks in duplicates, while HSR achieves it on a single ring network. PRP attaches a trailer at the end of each frame known as Redundancy Control Trailer (RCT) while HSR in the network attaches a HSR tag. In Fig. 2 is shown a PRP frame with redundancy control. IEC 62439-3 Ed. 1 defines RCT for 4 bytes tag while Ed. 2 extends it to 6 bytes. Ed.1 defines methods of supervision of frames while Ed. 2 lays down new guide lines to manage HSR. All HSR traffic gets recognized based on Ed. 2. The other notable difference in first and second edition of IEC 62439-3 is based on frame length. Ed. 1 had a restriction of 4 bytes for PRP but that restriction was lifted in Ed.2. Both PRP and HSR now have now 6 bytes in Ed. 2. Other conspicuous difference between Ed. 1 and Ed. 2 are based on sequence numbering of circulating frames, method of handling duplicate frames and node tables, clock synchronization [2].

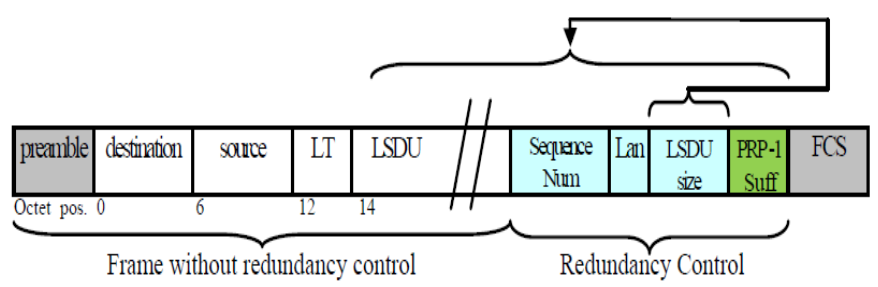

Fig. 3: PRP with RCT frame [2].

Figs. 3 and 4 show typical PRP and HSR tags respectively in the frames just before the addresses.

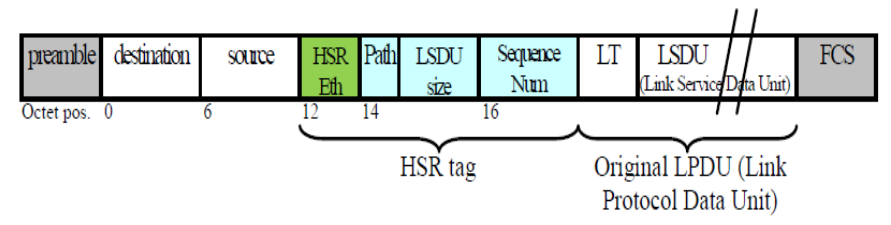

Fig. 4: HSR Frame with the tag at the front [2].

The disadvantage of HSR topology on the other hand is, it could accept undesirable circulating frames. The HSR network sends signals in two different directions within the ring. Each message in the loop carries a sequence number, which is accepted or rejected at the node depending on signal packets it carries [3]. Hence, HSR scheme has advantage of not only being a simple in structure with less hardware, but also accommodates higher traffic within the link that could lead to data clogging. This shortcoming is negated by applying bi-directional data communication channels which means, in case of a link failure, there are no stoppages to the data transfer circulating in healthier loop.

In this paper, a software based simulation is implemented in a laboratory environment using an Optimized Network Engineering Tool (OPNET) manufactured by Riverbed modeler offering simulations. The simulation result exhibits delay encountered and traffic load of data packets reaching out to various nodes in bits/sec based on these two seamless topologies. The frame stacks that carry Generic Object Oriented Substation Events (GOOSE) delivers real time and mission critical messages to the IEDs that exhibit latency and delay in 
the overall network. Hence, it is important to reduce the network traffic and bandwidth [4].

Summarizing the benefits of PRP and HSR topologies [57]:-

- Seamless protocol could be achieved using these topologies

- Availability of the SAS function through zero recovery time from a single link failure

- No time delay in the event of a fault due to transmission of frames in duplicates

- Easy coupling between PRP and HSR network to have best of both the features i.e. Combination of PRP and HSR, making the protection scheme robust, reliable and economical

- Tolerates any single network component failure

- Allows nodes not equipped for redundancy to operate by introducing Red boxes in the network

- Supports time synchronization

- Reduction in interoperability issue of different manufacturer products.

This paper is divided into 5 sections. Section I is for introduction which provides the background of the digital protection explaining the operation of PRP and HSR topologies, Section II deals with the node structures of PRP and HSR topologies. Section III provides the details of topologies in a substation environment, and IV details with the OPNET simulation results and discussion. Finally, the conclusion is presented in section $\mathrm{V}$.

\section{NODE STRUCTURES IN PRP AND HSR TOPOLOGIES}

\section{A) PRP Node Structure}

In a PRP node structure shown in Fig. 5, DANP has two Ethernet adapters but one Medium Access Control (MAC) and Internet Protocol (IP) address. Resilience is achieved by sending frames in two different directions in the ring. It provides seamless or bump-less redundancies which in other words, data is made available with zero delay during a link failure. In the event of a fault, any interruption which could prevent a frame from arriving at the node, shall cause other healthy frames to seek an alternative route, fulfilling the requirement of redundancy in the protection scheme. In this study, a station bus configuration has been considered to keep the structure simple. The timing requirement of station bus and process bus topologies are distinct, which underlines the protection redundancy requirement. In a station bus, delay up to a magnitude of 100-ms are tolerable for interlocking and trip, but for a reverse blocking, only 4-ms tolerance is acceptable as enumerated in IEC 61850-5 and exhibited in communication recovery time in Table-I.

In Fig. 5, a cross linked duplicate PRP network is shown in the form of a block diagram. It has two layers of redundancies with MAC and IP address present in each of the adapters. Furthermore, Link Redundancy Entity (LRE) act as a buffer between upper layer and ports. In a source node, LRE duplicates the frames, while at the destination node it monitors the packet flow in the duplicate loop. If a link or port is damaged, LRE shall continue to receive copies of the frames through an alternative path, while discarding the error frames. Additionally, in LRE, modification is achieved by the software and processor.

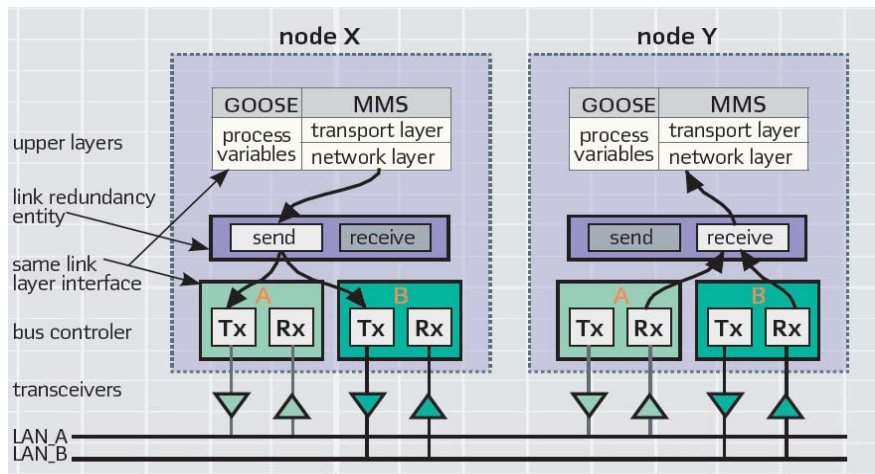

Fig. 5: Node structure of PRP [6].

\section{B) High Availability HSR Node Structure}

In a HSR topology, IEDs are daisy chained in a ring. Frames are sent in opposite directions at the same time in a loop. During a fault, error frames are discarded while healthier frames arrive at the destination node with zero recovery time, similar to the PRP topology choosing an alternative route. This ensures no down time in the network. As an example, when the unicast frames are sent in a loop, the frames broadcast messages to every node in the loop, with significant chances of getting acceptance at least by one IED. IEC 62439-3 stipulates in the event of a reduced traffic in the network, each node shall forward the frames within $5 \mu \mathrm{s}$. In other words, with duplicated frame messages travelling in opposite directions in the loop, frame messages are never lost. This feature in HSR topology enables frames are never lost and scheme offers total protection with zero-fault recovery time, in the event of a node or link failure. It restores SAS network to a healthy condition faster than a conventional system from clearing fault in a protection scheme perspective. However, the major disadvantage of the HSR topology is in duplication of messages within the ring, which could cause slowdown of frames due to data clogging in Ethernet traffic.

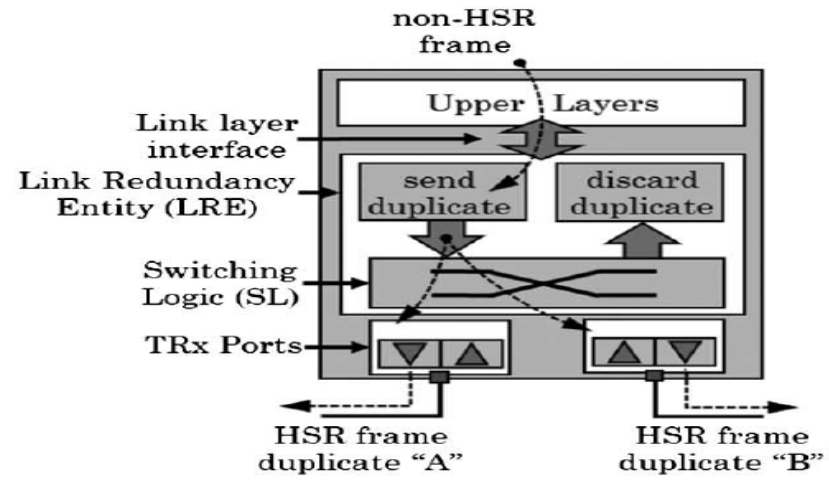

Fig. 6: Node structure of HSR [7].

In Fig. 6, a block diagram of HSR topology is shown which has a bridging logic between two ports $A$ and $B$ and vice versa. The duplication of frames are more pronounced in HSR than PRP. The disadvantage of HSR is duplicate detection of frames 
that could cause flooding in the ring structure. It is observed that in the bridging logic, clock synchronization of PRP and HSR relies on IEEE $1588 \mathrm{~V} 2$.

\section{Network Topologies IN A Station BUS CONFIGURATION}

Fig. 7 exhibits a Single Line Diagram (SLD) which shows a typical $132 / 22-\mathrm{kV}$ high voltage zone substation in a single bus configuration. Here, we have investigated the performance of a protection system based on PRP and HSR topology using OPNET simulator in a station bus configuration, having Ethernet ports on IEDs. In RSTP IEDs Red boxes are used transform it to PRP and HSR configuration.

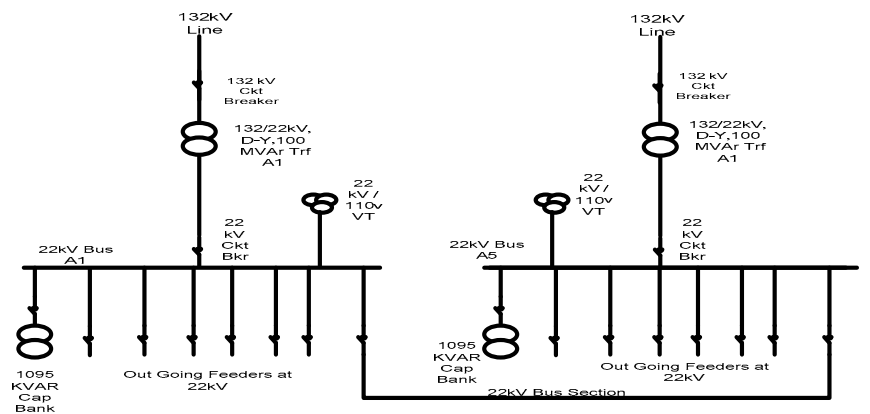

Fig. 7. The SLD of a 132/22-kV zone substation.

\section{A) Protection Scheme in a PRP Topology}

The IEDs in the block diagram shown in Fig. 4 are connected to switches in duplicated rings interlinked in a meshed structure. In this scheme, every IED has 2 layers of links providing seamless $\mathrm{N}-1$ redundancy.

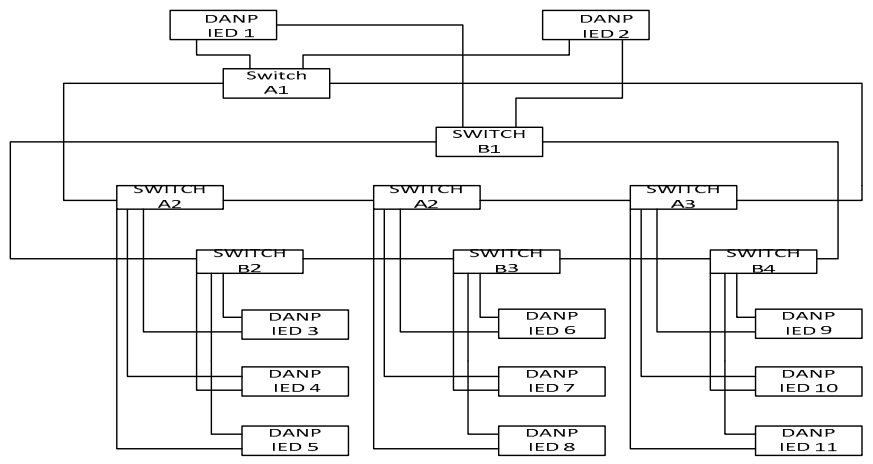

Fig. 8: PRP connection diagram.

\section{B) Protection Scheme in a HSR Topology}

In HSR topology, the nodes of the IEDs are either connected in DANP or SAN scheme via a Red box as shown in Fig. 9 in a ring structure. When two frames are sent out in opposite directions within the loop, the frame having error is blocked while the other reaches travelling on a healthier loop at its destination node, preventing loss of frames. The transmittal of frames in opposite directions, keep the communication channel in a high availability mode. Failure and error with frames makes the network vulnerable, which is eliminated by bi-directional communication.

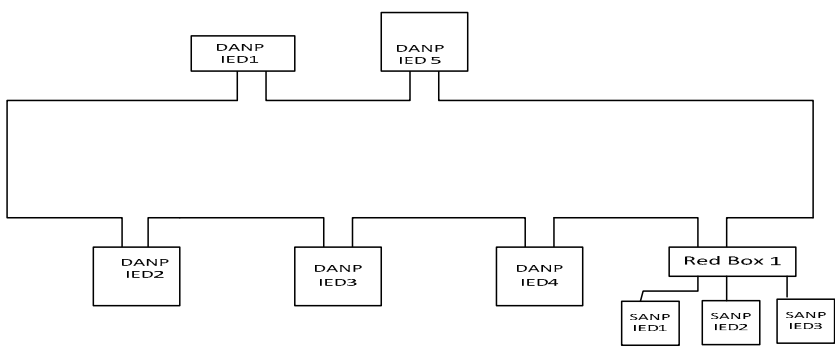

Fig. 9: HSR LAN in a single ring.

\section{Simulation Results AND Discussion}

\section{A) PRP Simulation}

An OPNET simulator is used to verify the performance of overall delay of a PRP network at the nodes of IEDs and switches. This simulation involved GOOSE traffic messages floating around in the duplicate local area network. Fig. 10 exhibits the Ethernet delay at the DANP nodes for an overall PRP connected protection scheme of a typical $132 / 22-\mathrm{kV}$ zone substation in a utility environment.

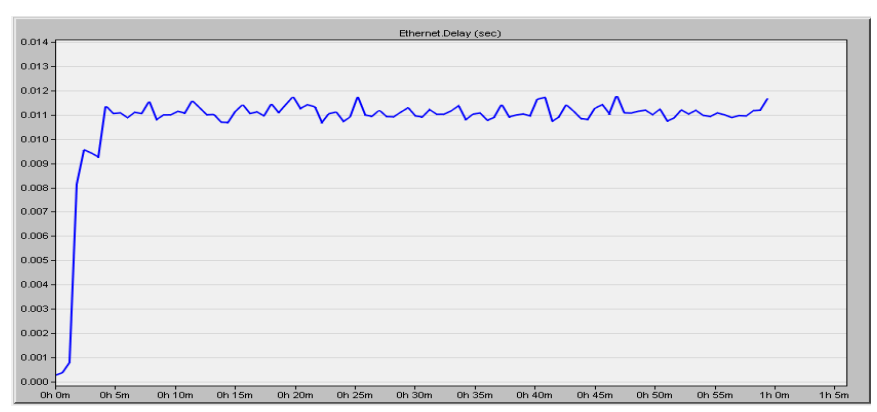

Fig. 10: Ethernet delays based on PRP topology.

Fig. 11 shows the overall Ethernet delay i.e., End-to-End delay in the topology which is encountered for all Ethernet connected IED devices and peripherals such as switches, media converters and Merging Units etc.

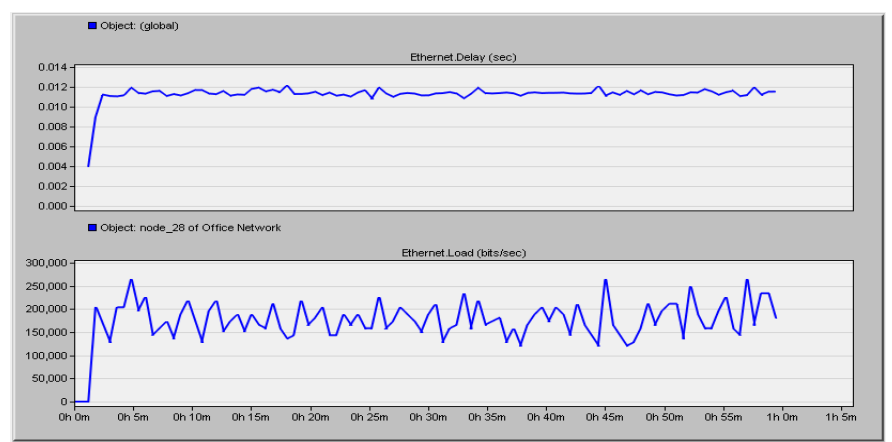

Fig. 11: Average Ethernet delay at nodes in a PRP topology vs overall Ethernet End to End delay.

\section{B) HSR Simulation}

Figs. 12 and 13 exhibit delay in Ethernet network at the DANP and SAN nodes for an overall HSR ring topology. Here, connected IEDs in the ring are HSR compatible devices but few SAN IEDs need Red box to support this topology. The reason for inserting Red boxes into the scheme is to prove the performance of SAN IEDs in a substation could be modified from an old structure to new without making these devices 
obsolete during protection upgrade from IEC 61850 to IEC 62439-3.

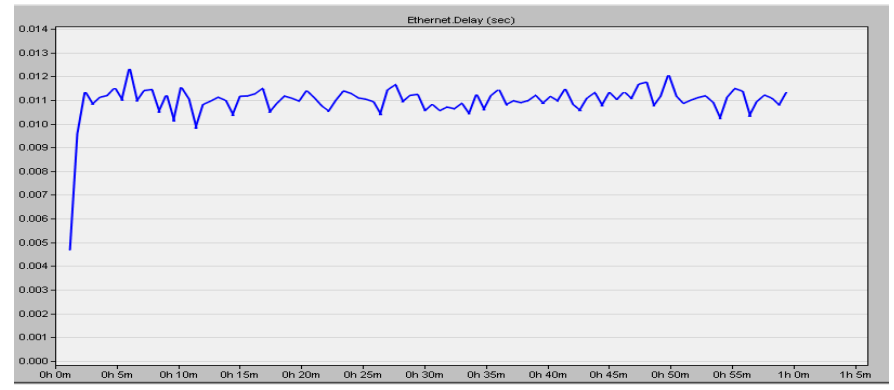

Fig. 12: Ethernet delays based on HSR topology.

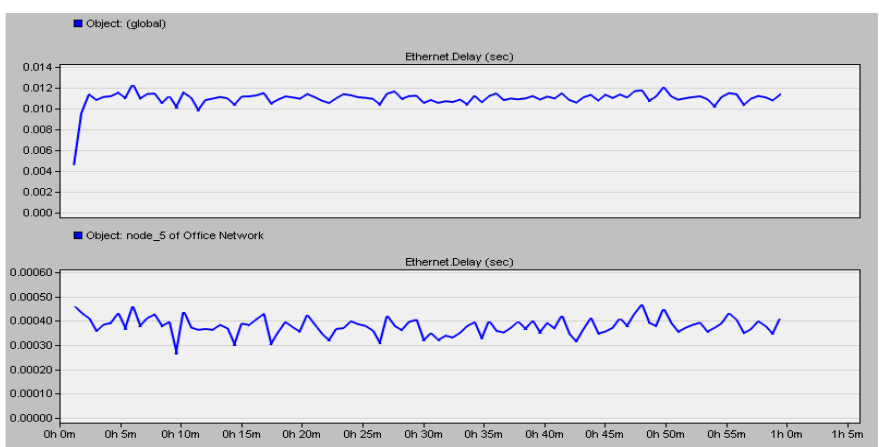

Fig. 13: Average Ethernet delays in a HSR topology node vs the overall delay.

The OPNET simulation for PRP and HSR topologies for performance evaluation were carried out with the following parameters as listed in Table-II.

Table-II: OPNET simulation for PRP and HSR topologies.

\begin{tabular}{|l|c|c|}
\hline & PRP & HSR \\
\hline Events & $11,450,473$ & $1,699,890$ \\
\hline Average Speed (events/sec) & $2,553,573$ & $1,699,890$ \\
\hline Time elapsed (sec) & 4 & 2 \\
\hline Duration of simulation (Hrs) & 1 & 1 \\
\hline DES Log & 6 entries & 6 entries \\
\hline
\end{tabular}

The OPNET simulation results indicate that the traffic delay at the nodes were slightly higher for HSR in comparison to PRP topology due to slow down of frames in the loop due to congestion of traffic routed via several nodes at different devices connected in a ring. The other notable conclusion inferred with respect to this simulation is, in terms of reliability, PRP offered better protection due to cross links and duplication of the ring having minimal chance of failure. PRP rings are virtually local area networks (LAN) that are resilient to faults, due to duplication of rings in the scheme. On the other hand, HSR topology having a simple architecture, with multiple IEDs in switching end nodes are effective in lesser complex digital protection network. The notable features in both these topologies i.e., PRP and HSR are, IEC 61850 devices having single port could be used in IEC 62439-3 inserting Red boxes and connected via fiber optic multiplexing adapters as shown in Fig. 5. This offers not only flexibility but could be upgraded to newer schemes without suffering from technology obsolescence.

\section{CONCLUSION}

Protection schemes in a substation require zero recovery time in the event of a fault. In other words, the circuit must provide zero recovery time to bounce back into active normal mode after suffering a failure. With the advancement of technology, it is possible to achieve these objectives using PRP and HSR topologies leveraging on IEC 62439-3 standard that offers failindependent protection scheme in a digital protection network.

This paper validates on a software platform the performance analysis of two digital network topologies in an Ethernet platform i.e. PRP and HSR based on IEC 62439-3 standard. The software simulation results were performed on a typical 132/22$\mathrm{kV}$ station bus architecture, which exhibited higher reliability and enhanced performance within a digital communication network. It validated the speed of frames arriving at nodes as per IEC 62439-3 and encouraged its application for future use within the utilities.

Both topologies promise enhanced fault tolerance strength in protection and redundancy. These topologies promise to be the future of automation and protection and it is recommended to be used in digital protection schemes within the utilities and industries.

\section{ACKNOWLEDGEMENT}

The authors gratefully acknowledge the valuable support and contribution made by Curtin University's industrial partners GE, CSE Universe, ABB, Schneider, ALSTOM, and Omicron.

\section{REFERENCES}

[1] J. A. Araujo, J. Lazaro, A. Astaloa, A. Zuloaga, and N. Moeira, "Duplicate and Circulating frames discard methods for PRP and HSR (IEC 62439-3)." in Proc. of the 2013 Electricity Distribution, CIRED 2013, DOI 10.1049/cp.2013.0836, Stockholm, Sweden.pl check all refs.

[2] C. Hoga,"Seamless Communication Redundancy of IEC 62439" in Proc. of The international Conference on Advanced Power System Automation and Protection 2011, DOI 10.1109/APAP.2011.6180451, Beijing, China.

[3] J. A. A raujo, J. Lazaro, A. Astaloa, A. Zuloaga, and A. Garcia, "PRP and HSR Version 1 (IEC 62439-3 Ed.2) Improvements and a Prototype implementation" in the Proc. of 2013 Industrial Electronics Society, IECON 2013, DOI 10.1109/IECON.2013.6699845, Vienna, Austria.

[4] G. Antonova, L.Frisk, and J. C. Tournier, "Communication Redundancy for Substation Automation" in Proc. of the $64^{\text {th }}$ Annual conference for Protective Engineers, 2012, DOI 10.1109/CPRE.2011.6035636, College Station, Tx, USA.

[5] International Standard IEC 62439-3, Edition 3.0, "Industrial Communication Networks High Availability automation networks - Part 3: Parallel Redundancy Protocol (PRP) and High-availability Seamless Redundancy (HSR)", 2012.

[6] H. Kirrmann, P. Rietmann, and S. Kunsman, "Standard IEC 61850 Network redundancy using 62439", Offprint of article in PAC World, Fall 2008.

[7] Y. M. Allawi, D. Lee, K. Lee, and J-K. K. Rhee. "Cost effective Topology design for HSR resilient Mesh Networks" in proc. of the Electricity Distribution (CIRED 2013), $22^{\text {nd }}$ International Conference an Exhibition, 10.1049/cp.2013.0836, Stockholm, Sweden. 